\title{
Signal-Processing Techniques to Reduce the Sinusoidal Steady-State Error in the FDTD Method
}

\author{
Levent Gürel, Senior Member, IEEE, and Uğur Oğuz
}

\begin{abstract}
Techniques to improve the accuracy of the finite-difference time-domain (FDTD) solutions employing sinusoidal excitations are developed. The FDTD computational domain is considered as a sampled system and analyzed with respect to the aliasing error using the Nyquist sampling theorem. After a careful examination of how the high-frequency components in the excitation cause sinusoidal steady-state errors in the FDTD solutions, the use of smoothing windows and digital low-pass filters is suggested to reduce the error. The reduction in the error is demonstrated for various cases.
\end{abstract}

Index Terms-Aliasing, digital filters, electromagnetic scattering, finite-difference time-domain, incident-field array, sampling, signal processing, smoothing windows.

\section{INTRODUCTION}

$\mathbf{T}$ HE finite-difference time-domain (FDTD) method has been used to solve a wide variety of problems in the area of computational electromagnetics during the last three decades [1]-[5], [14]. These problems include "frequency-domain" problems [2]-[13], where the excitation is a monochromatic time-harmonic source, in addition to truly "time-domain" problems containing multifrequency sources. Although the real power of the FDTD method lies in its ability to handle multifrequency signals, the simplicity of the method and its ability to easily model complicated inhomogeneities at no extra cost cause the method to become a preferable alternative for single-frequency problems. For instance, in order to compute the radar cross section (RCS) of an object at multiple frequencies, some researchers preferred to employ the FDTD method to solve several independent scattering problems, where the object is illuminated by a single-frequency sinusoidal signal in each solution.

In this paper, we will investigate the errors due to the propagation of the high-frequency components of signals that are introduced to the FDTD grid through user-defined excitations. Specifically, we will investigate the errors observed in the FDTD solutions when the excitation is sinusoidal. Errors in the form of nonphysical dc offsets, signal distortions, and added noise have been reported in the literature [10], [11], [13], [18] when sinusoidal excitation is used. Upon gaining a better understanding of these types of errors in Section II, techniques to reduce sinusoidal steady-state errors will be presented in Sections III and IV.

Manuscript received April 1, 1997; revised November 10, 1999.

The authors are with the Department of Electrical and Electronics Engineering, Bilkent University, Bilkent, Ankara, TR-06533 Turkey.

Publisher Item Identifier S 0018-926X(00)03266-X.

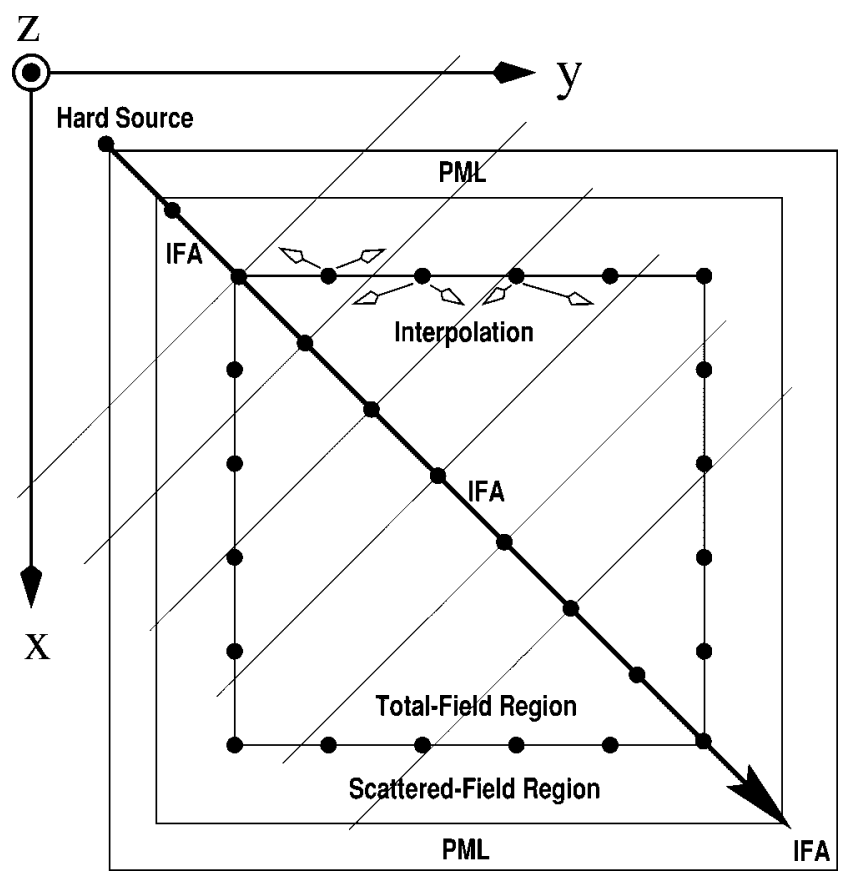

Fig. 1. The IFA excitation scheme in the separate-field formulation. The 1-D source grid (IFA) points in the direction of propagation, which is arbitrary and not necessarily confined to the $x-y$ plane as shown here. The incident-field values in the computational domain are interpolated from the two closest elements of the 1-D source grid (when linear interpolation is used).

The usefulness of the error-reducing techniques presented in this paper will be demonstrated using plane-wave excitations with sinusoidal time dependence. However, the applicability of these techniques are not limited to the plane-wave excitations; they are valid for any form of sinusoidal excitation. Indeed, since the plane waves are generated using an incident-field array (IFA) [4], [24], [25] (in a separate-field formalism [9], [10], [23]) in this work, even the plane waves are actually generated using "hard sources" and, thus, the error-reducing techniques are actually applied to finite-sized sources in the FDTD computations.

\section{SINUSOIDAL INCIDENT-WAVE EXCITATION}

When the FDTD method is used to investigate the sinusoidal steady-state response of an electromagnetic system, the usual way of supplying the excitation is to turn on a sinusoidal source at $t=0$ [8]. Then, a finite period of time, whose length depends on the specific problem, must pass before all the transients vanish and the steady-state is reached at every FDTD node. Furthermore, a few more half periods must pass before sinusoidal steady-state can be recognized and the required parameters can be extracted from the maxima of the field variables. A faster 


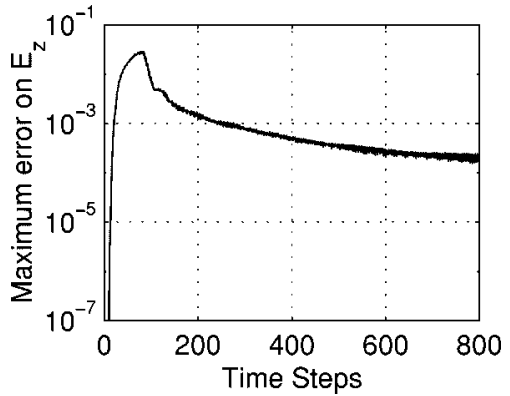

(a)

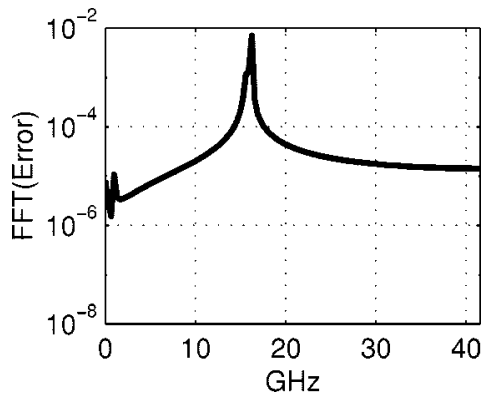

(b)

Fig. 2. Error results for the IFA excitation with no smoothing window, and no digital filter. (a) Maximum error on $E_{z}$ in the computational domain. (b) Frequency-domain representation of the error signal on $E_{z}$ at a particular point.

technique to extract the sinusoidal steady-state amplitude of a signal is outlined in [24, Appendix].

In addition to the transients that are related to the physics of the problem (such as multiple-scattering delays), the FDTD method also adds other transients to the solution as the problem is transformed from an analytical description to a computational representation. Some of these transients may be decaying very slowly or not decaying at all. Thus, these transients cause slowly-decaying or resident errors in the FDTD results. These computational transients (or errors) are controlled by the parameters of the FDTD method, including the representation of the excitation.

In order to separate these computational transients from the physical transients of the problem, FDTD simulations of the excitation and propagation of plane waves in homogeneous media are considered as shown in Fig. 1. A three-dimensional (3-D) empty computational domain composed of $30 \times 30 \times 30$ Yee cells and terminated by eight-cell-thick perfectly matched layer (PML) walls [15]-[17] is set up for this purpose. The PML walls are designed to have a theoretical normal reflection ratio $R(0)$ of $10^{-4}$ and parabolic conductivity profile. The space sampling period is $\Delta=0.625 \mathrm{~cm}$. The time step is selected at the Courant stability limit as $\Delta t=12.0281 \mathrm{ps}$. Separate-field formulation is employed with a total-field region of $18 \times 18 \times 18$ cells and a six-cell-thick scattered-field region. The incident plane-wave values are computed with the IFA scheme and fifth-order interpolation as described in [24] and [25]. The plane wave is incident at $\theta=90^{\circ}$ and $\phi=45^{\circ}$. The incident electric field is polarized in the $z$ direction and its amplitude is unity. The incident magnetic field is polarized in the direction of $\hat{x}-\hat{y}$. The time dependence of the incident plane wave is given by

$$
e(t)=u(t) \sin \left(2 \pi f_{0} t\right)
$$

where $f_{0}=1 \mathrm{GHz}$ and $u(t)$ is the unit step function. In other words, the sinusoidal excitation is started at $t=0$, similar to the earlier practice in this area [8]. Fig. 2(a) shows the maximum value of the error in the $E_{z}$ component over both the total-field and scattered-field domains at each time step. It is seen that the maximum error in the computational domain decreases very slowly and does not reach its steady-state value after 800 time steps, which correspond to about 10 periods at $1 \mathrm{GHz}$.

In order to have a better understanding of the nature of the error, Fig. 2(b) shows the frequency spectrum of the error in the $E_{z}$ component at the first cell the incident wave touches the total-field domain. With the chosen FDTD time-step $(\Delta t)$ value, frequencies up to about $41.5 \mathrm{GHz}$ can be resolved, thus the spectrum of the error signal is computed (via FFT) from dc to $41.5 \mathrm{GHz}$.

Upon examining the error plots of Fig. 2, the following points become evident.

1) The transient error is yet to diminish even after 800 time steps.

2) The error is falling very slowly with respect to time.

3) The error is a high-frequency error peaking around 16-17 GHz as seen in Fig. 2(b), despite the fact that the input signal (the plane-wave excitation) is a sinusoidal at $1 \mathrm{GHz}$. Fig. 2(a) also displays rapid oscillations, which clearly indicate the high-frequency nature of the error.

The third observation in the above concerning the high-frequency content of the error signal can be explained by realizing that the abrupt application of a sinusoidal signal is equivalent to multiplication by a step function as in (1). The Fourier transform of the signal $e(t)$ in (1) is given by

$$
\begin{gathered}
E(\omega)=\frac{1}{2}\left[j \pi \delta\left(\omega+\omega_{0}\right)-j \pi \delta\left(\omega-\omega_{0}\right)\right. \\
\left.+\frac{1}{\omega+\omega_{0}}-\frac{1}{\omega-\omega_{0}}\right] .
\end{gathered}
$$

The signal itself and the spectrum thereof are shown in Fig. 3. This signal, which clearly contains frequency components above $41.5 \mathrm{GHz}$, is sampled at a rate of $f_{s}=83 \mathrm{GHz}$ and, consequently, is aliased with an aliasing interval of $\omega_{s}=2 \pi f_{s}$. Thus, in terms of the frequency spectrum of $e(t)$ as defined in (2), the frequency spectrum of the sampled signal is given by

$$
E_{s}(\omega)=\frac{1}{T_{s}} \sum_{n=-\infty}^{\infty} E\left(\omega-n \omega_{s}\right)
$$

where $T_{s}=1 / f_{s}$. The error due to aliasing (AE: $\underline{\text { Aliasing Error) }}$ is defined as

$$
\mathrm{AE}=\left|\frac{1}{T_{s}} \sum_{\substack{n=-\infty \\ n \neq 0}}^{\infty} E\left(\omega-n \omega_{s}\right)\right|
$$

in the frequency range from dc to $f_{s} / 2=41.5 \mathrm{GHz}$, which is the highest frequency component that can be resolved by sampling at a rate of $f_{s}=83 \mathrm{GHz}$ due to the Nyquist sampling theorem [19], [20]. Fig. 4(a) depicts the aliasing error as defined in (4). Thus, the frequency components of the signal shown in Fig. 3 above $41.5 \mathrm{GHz}$ contaminate the frequency 


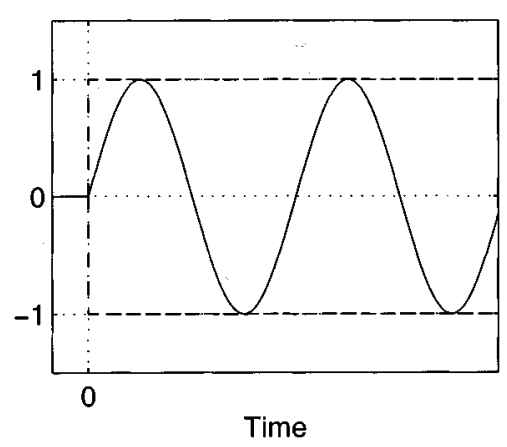

(a)

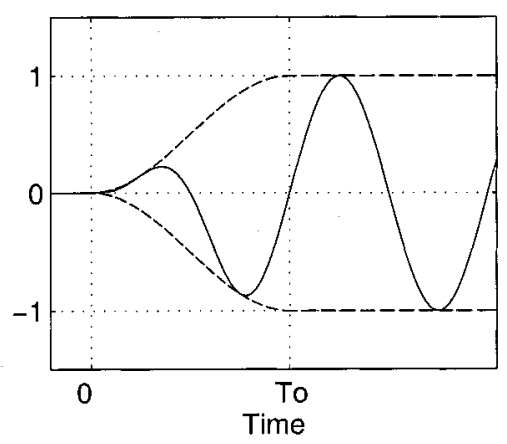

(c)

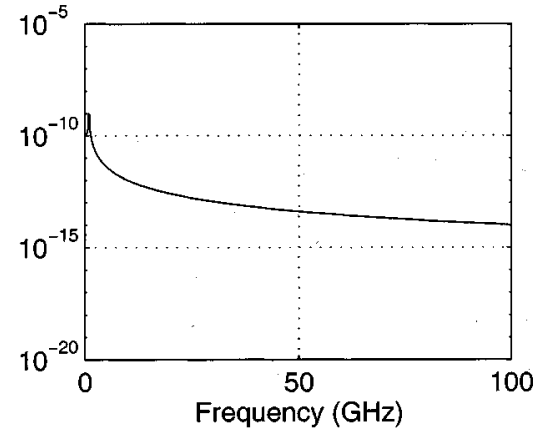

(b)

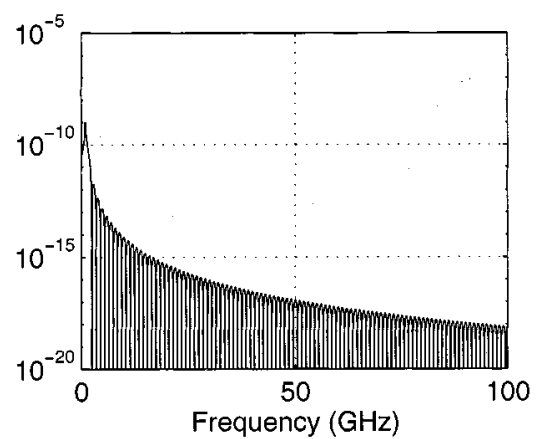

(d)

Fig. 3. (a) Time and (b) frequency representations of the input signal in (1). No smoothing window is applied to the input. The sinusoidal signal is multiplied by a step function at $t=0,(\mathrm{c})$ time, and (d) frequency representations of the input signal in (6). A Hanning window with length $L=T_{0}$ is used for smoothing at $t=0$.

band below $41.5 \mathrm{GHz}$ due to aliasing. It can be assumed that it is these high-frequency components that cause the slowly-decaying errors seen in Fig. 2. This assumption will be tested in the following sections by using techniques to reduce the high-frequency components of the excitation and observing the effect of this reduction on the aliasing error [as in Fig. 4(a)] and the error in the FDTD computations (as in Fig. 2).

\section{USE OF SMOOTHING WINDOWS}

One way to reduce the high-frequency components of the signal shown in Fig. 3(a) is to multiply it by a window that is smoother than the rectangular window (step function). A triangular (Bartlett), Hanning (Hann), Hamming, or Blackman window [19], [21] can be used for this purpose. The triangular window is also used by Kunz and Luebbers [5]. In this section, we will investigate the effects of other smoothing windows on the aliasing and the FDTD errors.

The Hanning, Hamming, and Blackman windows are defined as (5), shown at the bottom of the page. Fig. 3(c) and (d) shows a signal

$$
e_{w}(t)=w(t) \sin \left(2 \pi f_{0} t\right)
$$

and its frequency spectrum, respectively, where $w(t)$ is a Hanning window with $L=T_{0}=1 / f_{0}$. Comparing Fig. 3(d) to (b), we observe that $e_{w}(t)$ has smaller high-frequency components than $e(t)$. Next, we will investigate how the aliasing error as defined in (4) will change using various smoothing windows.

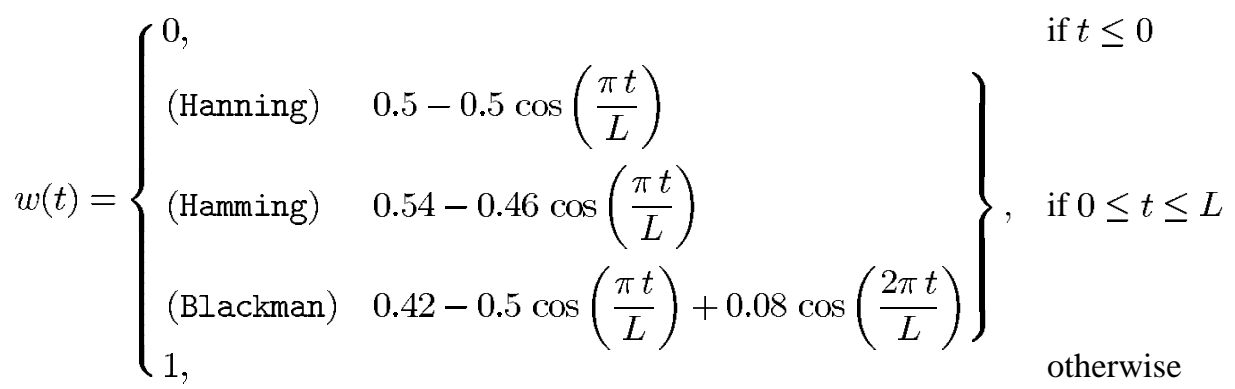




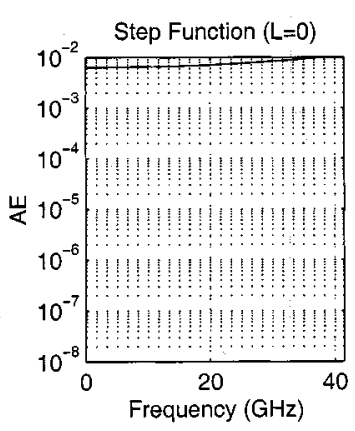

(a)

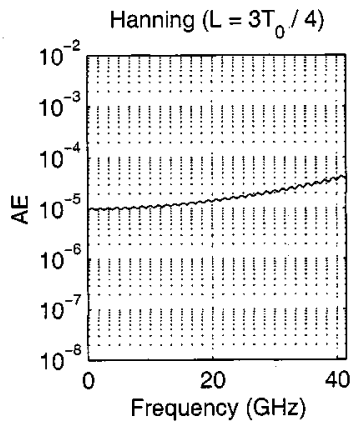

(d)

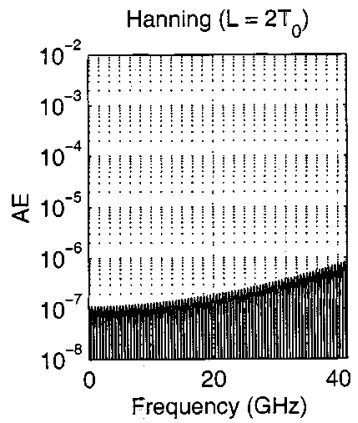

(g)

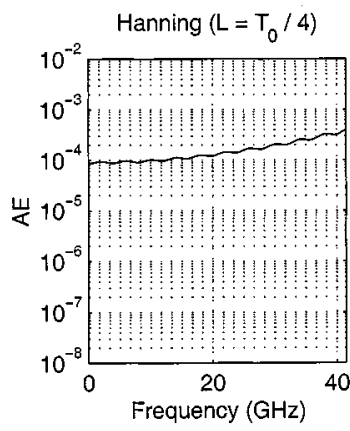

(b)

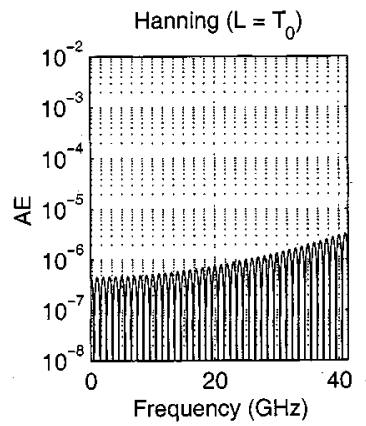

(e)

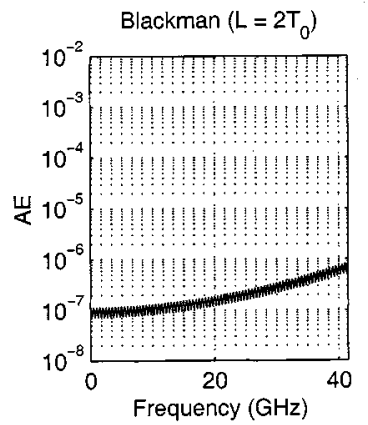

(h)

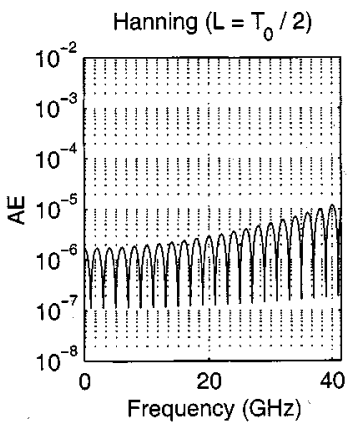

(c)

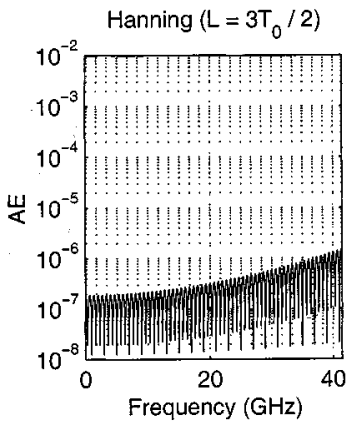

(f)

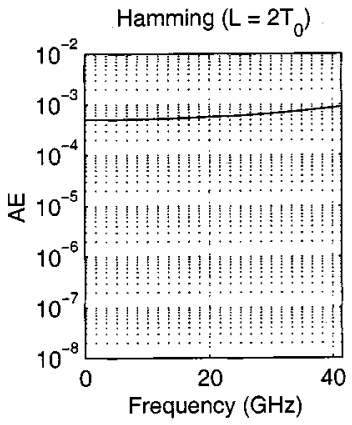

(i)

Fig. 4. (Continued.) Aliasing errors (AE) versus frequency for smoothing windows: (a) no smoothing window $(L=0)$. (b) Hanning with $L=T_{0} / 4$. (c) Hanning with $L=T_{0} / 2$. (d) Hanning with $L=3 T_{0} / 4$. (e) Hanning with $L=T_{0}$, (f) Hanning with $L=3 T_{0} / 2$. (g) Hanning with $L=2 T_{0}$. (h) Blackman with $L=2 T_{0}$. (i) Hamming with $L=2 T_{0}$.

As in (4), the aliasing error ( $\mathrm{AE}$ ) for windowed signals is defined by

$$
\begin{array}{r}
\mathrm{AE}=\left|\frac{1}{T_{s}} \sum_{\substack{n=-\infty \\
n \neq 0}}^{\infty} E_{w}\left(\omega-n \omega_{s}\right)\right| \\
=\left|\frac{j}{2 T_{s}} \sum_{\substack{n=-\infty \\
n \neq 0}}^{\infty}\left[W\left(\omega-n \omega_{s}+\omega_{0}\right)-W\left(\omega-n \omega_{s}-\omega_{0}\right)\right]\right|
\end{array}
$$

where $E_{w}(\omega)$ and $W(\omega)$ are the Fourier transforms of the windowed signal $e_{w}(t)$ and the windowing function $w(t)$, respectively. The AE for Hanning windows of lengths $L=T_{0} / 4, T_{0} / 2$, $3 T_{0} / 4, T_{0}, 3 T_{0} / 2$, and $2 T_{0}$ are shown in Fig. $4(\mathrm{~b})-(\mathrm{g})$. Although the $\mathrm{AE}$ is generally reduced as the window length increases, note that it is lower for the case of $L=T_{0} / 2$ than for $L=3 T_{0} / 4$. Fig. 4(h) shows that the performance of the Blackman window of length $L=2 T_{0}$ is very close to the performance of the Hanning window of the same length, shown in Fig. 4(g). In fact, this is the case for all Blackman windows with lengths $L=T_{0} / 4$, $T_{0} / 2,3 T_{0} / 4, T_{0}, 3 T_{0} / 2$, and $2 T_{0}$. Only the $L=2 T_{0}$ case is shown in Fig. 4, since the others perform very similar to the Hanning windows. The performance of the Blackman window with length $L=T_{0} / 2$ is better than the Blackman window with length $L=3 T_{0} / 4$, similar to the Hanning windows. In Fig. 4, only the $L=2 T_{0}$ case is shown for the Hamming windows. When Fig. 4(i) is compared to Fig. 4(a), it can be deduced that the Hamming window reduces the $\mathrm{AE}$, like the other window types. However, the Hamming window's performance does not change with the window length. The $\mathrm{AE}$ results for $L=T_{0} / 4$, $T_{0} / 2,3 T_{0} / 4, T_{0}$, and $3 T_{0} / 2$ are very close to the results for $L=2 T_{0}$. That is, no improvement is obtained by increasing the length of the Hamming window. This is probably due to the 


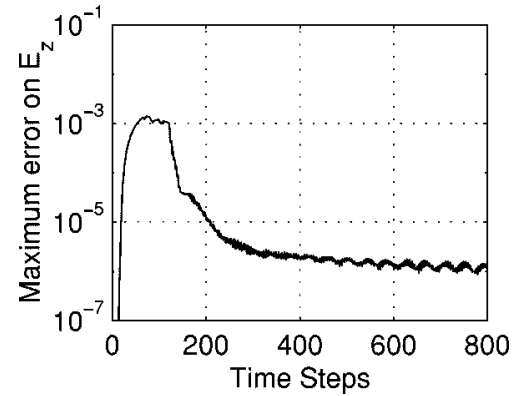

(a)

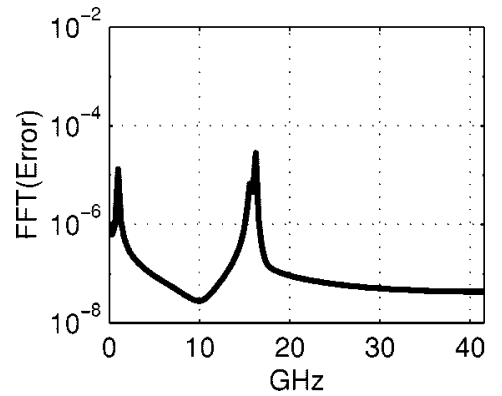

(b)

Fig. 5. Error results for the IFA excitation with Hanning window of length $L=T_{0} / 2$ : (a) maximum error on $E_{z}$ in the computational domain and (b) frequency-domain representation of the error signal on $E_{z}$ at a particular point.

finite jump of the Hamming window at $t=0$. This jump prevents the smoothing at $t=0$ and induces high-frequency components with larger amplitudes than the Hanning and Blackman windows do.

In order to investigate the effects of the smoothing windows on the FDTD error, a number of simulations are performed with the empty computational domain setup described in the previous section. The results for the Hanning windows of lengths $L=T_{0} / 2, T_{0}$, and $2 T_{0}$ are shown in Figs. 5-7, respectively. All of these results show great improvement with respect to the results obtained with no smoothing, shown in Fig. 2. Figs. 5(a), 6(a), and 7(a) show that the steady-state error level decreases as the window length is increased. In Fig. 5(b), it is observed that the dominant frequency component of the error signal is around $16-17 \mathrm{GHz}$ for the Hanning window of length $T_{0} / 2$. This high-frequency component is suppressed with longer Hanning windows, as shown in Figs. 6(b) and 7(b). Fig. 8 shows the steady-state error level with respect to the length of the Hanning window. A comparison of Figs. 4 and 8 reveals the parallelism between how the window length influences the $A E$ and the overall FDTD error, respectively, including the local minima of both types of errors for $L=T_{0} / 2$.

\section{USE OF Digital FiLTERS}

The smoothing windows of the previous section reduce the high-frequency components of the input signal. This helps to increase the accuracy since the FDTD algorithm cannot properly handle the sampled and, hence, aliased signals containing high-frequency components.

The goal of suppressing the high-frequency components of the input signals can also be achieved by using a low-pass filter. However, note that in order to prevent the aliasing, a low-pass

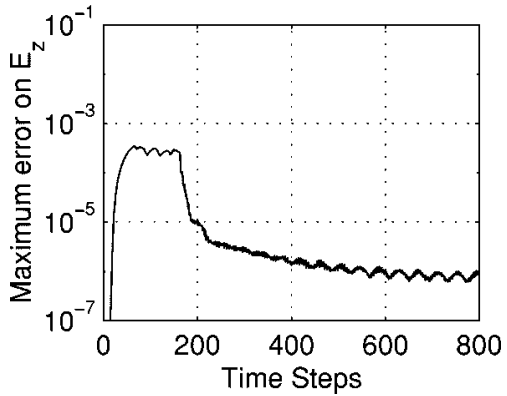

(a)

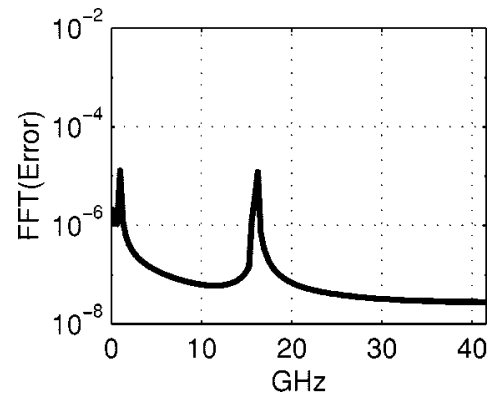

(b)

Fig. 6. Error results for the IFA excitation with Hanning window of length $L=T_{0}$ : (a) maximum error on $E_{z}$ in the computational domain and (b) frequency-domain representation of the error signal on $E_{z}$ at a particular point.

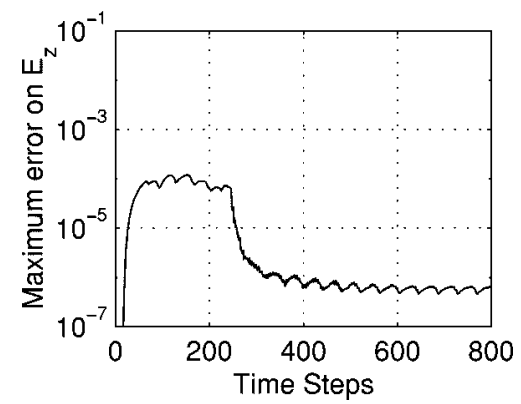

(a)

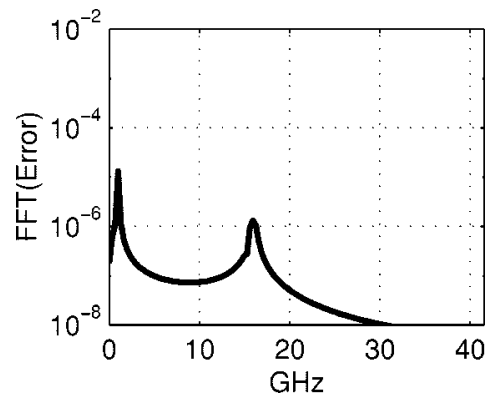

(b)

Fig. 7. Error results for the IFA excitation with Hanning window of length $L=2 T_{0}$ : (a) maximum error on $E_{z}$ in the computational domain and (b) frequency-domain representation of the error signal on $E_{z}$ at a particular point.

filter should be used before the sampling of the input signal. That would require an analog filter, which would be difficult to implement and would increase the incident-field computation time enormously. Instead, a digital low-pass filter can be used on the already sampled input signal before it is fed into the hard source of the IFA. Note that a digital filter used after the sampling cannot prevent the aliasing. Nevertheless, it still reduces 


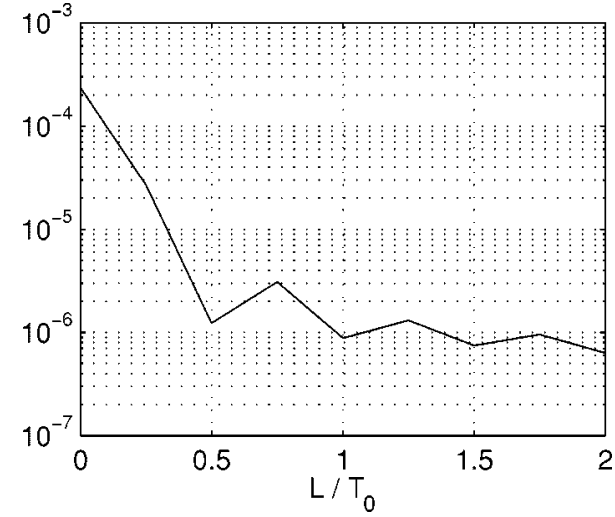

Fig. 8. Steady-state error level versus length of the Hanning window used for smoothing in terms $T_{0}$-the period of the incident wave.

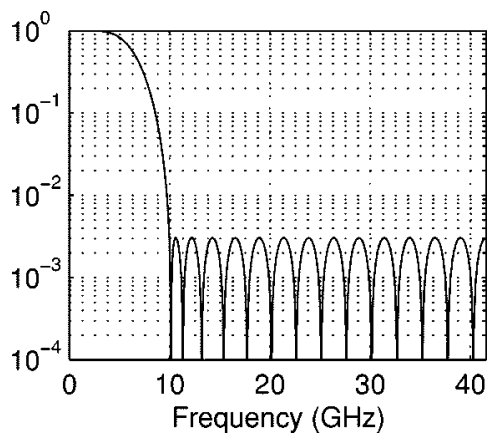

Fig. 9. The frequency response of the digital filter.

the FDTD error since it reduces the high-frequency input to the computational domain.

The frequency response of the 32-point digital filter [26], [27], [28] used in the FDTD simulations is shown in Fig. 9. This filter has unity gain and $10-\mathrm{GHz}$ cutoff. Figs. 10 and 11 show the error plots obtained by using this filter on two input signals: 1) with no smoothing window and 2) with Hanning window of length $L=T_{0} / 2$, respectively. A comparison of Figs. 2 and 10 shows the improvement in the error results when the input signal in (1) is passed through the filter before it is fed into the hard source. A similar comparison can be made for Figs. 5 and 11. The steady-state error is decreased below the $10^{-6}$ level, as shown in Fig. 11(a). Fig. 11(b) shows that the component of the error signal around $16-17 \mathrm{GHz}$ is significantly reduced by using the filter together with the Hanning window. The dominant frequency component of the error in Fig. 11(b) is $1 \mathrm{GHz}$, which is the operating frequency of the incident wave. The amplitude of the 1-GHz component of the error cannot be reduced by using smoothing windows or filters since it is produced by the numerical dispersion. Examining the Figs. 5(b), 6(b), 7(b), 10(b), and 11(b) carefully, it can be observed that the amplitude of the $1-\mathrm{GHz}$ component of the error remains the same regardless of the smoothing window or the filter used. Thus, the $1-\mathrm{GHz}$ component constitutes a threshold for the error level.

Comparing Figs. 2(a) and 11(a), we note that, in addition to reducing the level of the steady-state error considerably, the use of smoothing windows and filters also helps shorten the transient period required to reach the steady state. However, we also

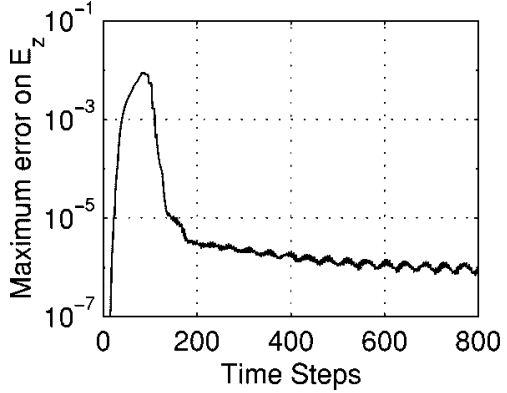

(a)

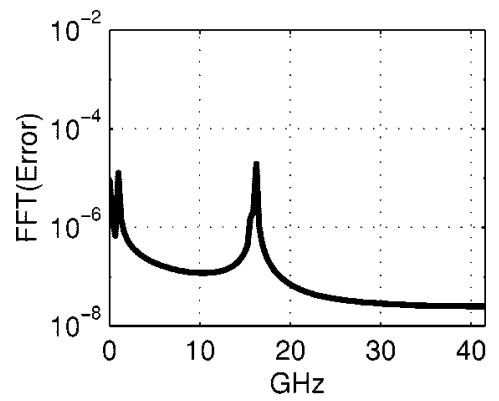

(b)

Fig. 10. Error results for the IFA excitation with the digital filter, but no smoothing window: (a) maximum error on $E_{z}$ in the computational domain and (b) frequency-domain representation of the error signal on $E_{z}$ at a particular point.

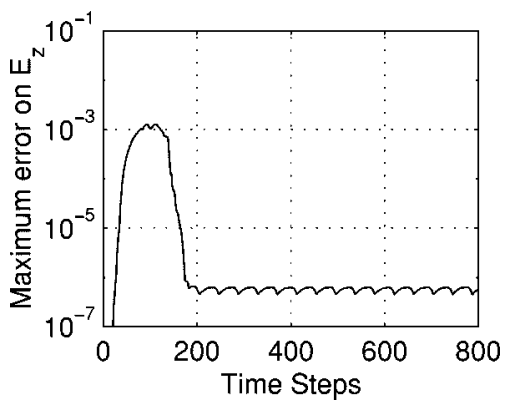

(a)

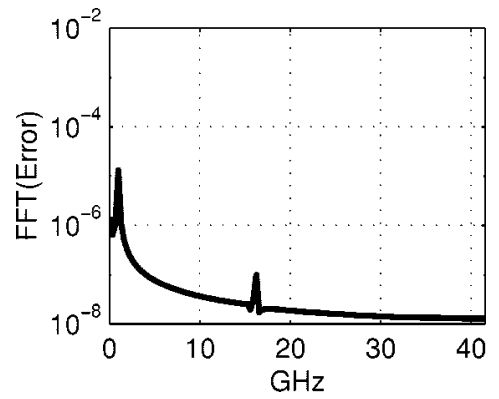

(b)

Fig. 11. Error results for the IFA excitation with the Hanning window of length $L=T_{0} / 2$ and also passed through the digital filter: (a) maximum error on $E_{z}$ in the computational domain and (b) frequency-domain representation of the error signal on $E_{z}$ at a particular point.

note that these calculations are performed in an empty computational domain and that the duration of the transient period will be mostly determined by the size of the inhomogeneity in FDTD calculations involving large objects. 
TABLE I

MAXimum STEAdy-STATE ERROR LEVELS ON THE AMPLITUdE OF $E$, AT NORMAL INCIDENCE

\begin{tabular}{l|c}
\hline IFA excitation scheme using ... & Error \\
\hline \hline No smoothing window or filter & $1.0 \times 10^{-3}$ \\
\hline A digital filter & $0.3 \times 10^{-5}$ \\
\hline A half-period long Hanning window & $0.4 \times 10^{-5}$ \\
\hline Hanning window and digital filter together & $1.2 \times 10^{-8}$ \\
\hline
\end{tabular}

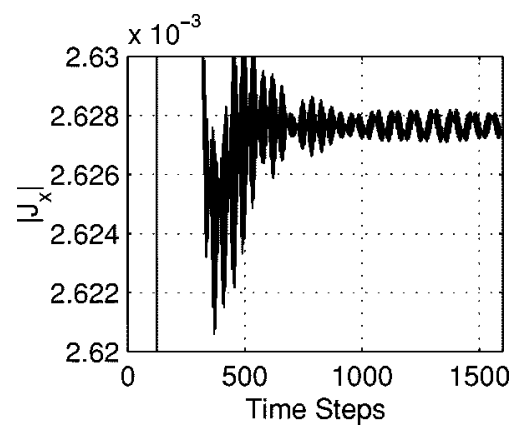

(a)

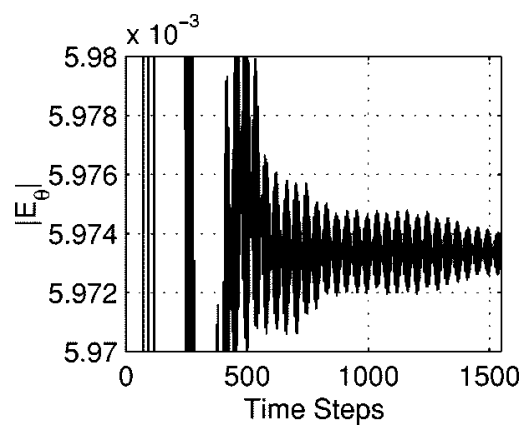

(b)

Fig. 12. Results for the metal-plate scattering problem. The incident fields are computed with the IFA excitation using no smoothing window or digital filter. (a) Amplitude of the induced current $J_{x}$ at a particular point. (b) Amplitude of the far-zone electric-field component $E_{\theta}$ in the direction of $\theta=90^{\circ}, \phi=$ $-45^{\circ}$.

\section{OTHER INTERESTING CASES}

\section{A. Normal Incidence}

The special case of normal incidence is obtained when the direction of the incident wave is $\pm x, \pm y$, or $\pm z$. For such special cases, the FDTD algorithm may generate exactly zero error, which was defined in the previous sections as the difference of the total-field and incident-field values. However, this does not mean that the total-field signal is free of errors in the sense that it is exactly the same as the desired perfect sinusoid. The reasons behind this are explained in [25], Section VI.

Table I uses a definition of the error that is different from that of the previous sections to present the error in the case of normal incidence. The error is defined as the maximum difference of the amplitude (computed at every time step using the method outlined in [24, Appendix]) of the total-field signal from unity for steady state (after 500 time steps).

The nonzero error presented on the first line of Table I, when no smoothing window or digital filter is applied to the input,

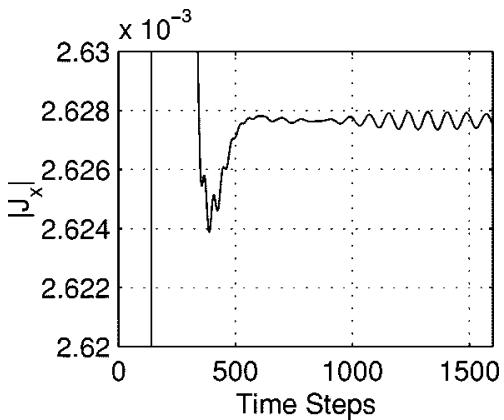

(a)

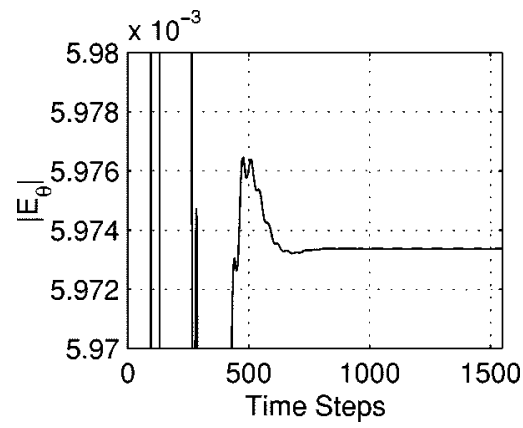

(b)

Fig. 13. Results for the metal-plate scattering problem. The incident fields are computed with the IFA excitation using a digital filter, but no smoothing window. (a) Amplitude of the induced current $J_{2}$ at a particular point. (b) Amplitude of the far-zone electric-field component $E_{\theta}$ in the direction of $\theta=90^{\circ}, \phi=-45^{\circ}$.

suggests that there is still room for improvement. Table I shows that the error level is decreased by about two orders when either a digital filter or a Hanning window of length $L=T_{0} / 2$ is used, and by another three orders when both of them are used.

\section{B. Scattering from a Patch}

The problem of scattering from a square metal plate of size $20 \times 1 \times 20$ Yee cells is used to demonstrate the effects of reducing the high-frequency components of the input. The plate lies on the $x-z$ plane, in the middle of a computational domain consisting of $40 \times 20 \times 40$ cells, which is divided into a total-field region of $28 \times 8 \times 28$ Yee cells and a six-cell-thick scattered-field region. The incident plane wave is identical to the one in Section II. The center of the bright face of the plate is called "the origin" of the 3-D grid and given the index $(0,0,0)$. Figs. 12(a) and 13(a) show the $x$ component of the induced surface current observed at the point $(-5,0,-5)$ on the bright face of the plate.

Figs. 12(b) and 13(b) present the $\theta$ component of the far-zone electric field, $E_{\theta}$, which is extrapolated at a far-zone point, $(r, \theta, \phi)=\left(2000 \Delta, 90^{\circ}, 225^{\circ}\right)$. A time-domain far-zone extrapolation scheme [22] is used for this purpose. The integration surface used for the far-zone transformation is a parallelepiped located two cells out of the total field/scattered field interface.

The results shown in Fig. 12 are obtained by using the input given in (1); that is, no smoothing windows or filters are used to reduce the high-frequency components of the input. Fig. 13 shows the results obtained when the digital filter in Fig. 9 is applied to the input in Fig. 12. The oscillations in the final periods of the amplitude levels can be regarded as convergence errors, 
since the amplitudes are expected to converge to a final value as the transients die. Comparing Figs. 12 and 13, it is observed that the convergence error is reduced in the near-field and far-field signals by using a digital filter. The results obtained by using a Hanning window of length $T_{0} / 2$, alone and together with the filter, are not presented here since they are very close to the results shown in Fig. 13.

\section{CONCLUSIONS}

In this paper, we have demonstrated that the high-frequency components present in the input signal have adverse effects on the accuracy of the FDTD calculations. By considering the FDTD computational domain as a sampled system, we have clearly identified and discussed the origins of the FDTD errors for sinusoidal excitations using the Nyquist sampling theorem and the concept of the aliasing error. The use of smoothing windows and digital low-pass filters in reducing the error has been demonstrated. Using these techniques, it has been possible to reduce both the sinusoidal steady-state FDTD error by more than two orders of magnitude and shorten the transient period to reach the steady state. The amplitude and phase calculations of sampled sinusoidal signals are performed by using a fast two-point extraction technique, as outlined in [24, Appendix].

\section{REFERENCES}

[1] K. S. Yee, "Numerical solution of initial boundary value problems involving Maxwell's equations in isotropic media," IEEE Trans. Antennas Propagat., vol. AP-14, pp. 302-307, Apr. 1966.

[2] A. Taflove, "Review of the formulation and applications of the finite-difference time-domain method for numerical modeling of electromagnetic wave interactions with arbitrary structures," Wave Motion, vol. 10, no. 6, pp. 547-582, 1988.

[3] A. Taflove and K. R. Umashankar, "Review of FD-TD numerical modeling of electromagnetic wave scattering and radar cross section," Proc. IEEE, vol. 77, pp. 682-699, May 1989.

[4] A. Taflove, Computational Electrodynamics: The Finite-Difference Time-Domain Method. Boston, MA: Artech House, 1995.

[5] K. S. Kunz and R. J. Luebbers, The Finite Difference Time Domain Method for Electromagnetics. Boca Raton, FL: CRC, 1993.

[6] A. Taflove and M. E. Brodwin, "Numerical solution of steady-state electromagnetic scattering problems using the time-dependent Maxwell's equations," IEEE Trans. Microwave Theory Tech., vol. MTT-23, pp. 623-630, Aug. 1975.

[7] _ - "Computation of the electromagnetic fields and induced temperatures within a model of the microwave irradiated human eye," IEEE Trans. Microwave Theory Tech., vol. MTT-23, pp. 888-896, Nov. 1975.

[8] A. Taflove, "Application of the finite-difference time-domain method to sinusoidal steady-state electromagnetic-penetration problems," IEEE Trans. Electromagn. Compat., vol. EMC-22, pp. 191-202, Feb. 1980.

[9] G. Mur, "Absorbing boundary conditions for the finite-difference approximation of the time-domain electromagnetic-field equations," IEEE Trans. Electromagn. Compat., vol. EMC-23, pp. 377-382, Apr. 1981.

[10] K. R. Umashankar and A. Taflove, "A novel method to analyze electromagnetic scattering of complex objects," IEEE Trans. Electromagn. Compat., vol. EMC-24, pp. 397-405, Apr. 1982.

[11] A. Taflove and K. Umashankar, "Radar cross section of general three-dimensional scatterers," IEEE Trans. Electromagn. Compat., vol. EMC-25, pp. 433-440, Apr. 1983.

[12] A. Taflove, K. R. Umashankar, and T. G. Jurgens, "Validation of FD-TD modeling of the radar cross section of three-dimensional structures spanning up to nine wavelengths," IEEE Trans. Antennas Propagat., vol. AP-33, pp. 662-666, June 1985.

[13] G. J. A. van Gennip, "Theory and applications of the three-dimensional finite-difference time-domain method," TNO Phys. Electron. Lab., The Hague, The Netherlands, Tech. Rep. FEL-92-B190, June 1992.
[14] K. L. Shlager and J. B. Schneider, "A selective survey of the finite-difference time-domain literature," IEEE Antennas Propagat. Mag., vol. 37, pp. 39-56, Apr. 1995.

[15] J.-P. Bèrenger, "A perfectly matched layer for the absorption of electromagnetics waves," J. Comput. Phys., vol. 114, no. 1, pp. 185-200, 1994.

[16] _ - "Perfectly matched layer for the FDTD solution of wave-structure interaction problems," IEEE Trans. Antennas Propagat., vol. 44, pp. 110-117, Jan. 1996.

[17] — "Three-dimensional perfectly matched layer for the absorption of electromagnetic waves," J. Comput. Phys., vol. 127, pp. 363-379, 1996.

[18] C. M. Furse, S. P. Mathur, and O. P. Gandhi, "Improvements to the finitedifference time-domain method for calculating the radar cross section of a perfectly conducting target," IEEE Trans. Microwave Theory Tech., vol. 38, pp. 919-927, July 1990.

[19] A. V. Oppenheim and R. W. Schafer, Discrete-Time Signal Processing. Englewood Cliffs, NJ: Prentice-Hall, 1989.

[20] R. A. Gabel and R. A. Roberts, Signals and Linear Systems. New York: Wiley, 1973

[21] F. J. Harris, "On the use of windows for harmonic analysis with the discrete Fourier transform," Proc. IEEE, vol. 66, pp. 51-83, Jan. 1978.

[22] R. J. Luebbers, K. S. Kunz, M. Schneider, and F. Hunsberger, "A finite-difference time-domain near zone to far zone transformation," IEEE Trans. Antennas Propagat., vol. 39, pp. 429-433, Apr. 1991.

[23] D. E. Merewether, R. Fisher, and F. W. Smith, "On implementing a numeric Huygen's source scheme in a finite difference program to illuminate scattering bodies," IEEE Trans. Nucl. Sci., vol. 27, pp. 1829-1833, June 1980.

[24] U. Oğuz and L. Gürel, "Interpolation techniques to improve the accuracy of the incident-wave excitations in the FDTD method," Radio Sci., vol. 32, no. 6, pp. 2189-2199, Nov./Dec. 1997.

[25] U. Oğuz, L. Gürel, and O. Arıkan, "An efficient and accurate technique for the incident-wave excitations in the FDTD method," IEEE Trans. Microwave Theory Tech., vol. 46, pp. 869-882, June 1998

[26] L. R. Rabiner, J. H. McClellan, and T. W. Parks, "FIR digital filter design techniques using weighted Chebyshev approximations," Proc. IEEE, vol. 63, pp. 595-610, Apr. 1975

[27] T. W. Parks and C. S. Burrus, Digital Filter Design. New York: Wiley, 1987.

[28] J. N. Little and L. Shure, MATLAB Signal Processing Toolbox User's Guide. Natick, MA: MathWorks, 1992.

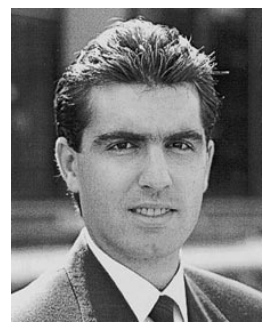

Levent Gürel (S'87-M'92-SM'97) was born in İzmir, Turkey, in 1964. He received the B.Sc. degree from the Middle East Technical University (METU), Ankara, Turkey, in 1986, and the M.S. and $\mathrm{Ph} . \mathrm{D}$. degrees from the University of Illinois at Urbana-Champaign (UIUC), in 1988 and 1991, respectively, all in electrical engineering.

He joined the Thomas J. Watson Research Center of the International Business Machines Corporation, Yorktown Heights, NY, in 1991, where he worked as a Research Staff Member on the electromagnetic compatibility (EMC) problems related to electronic packaging on the use of microwave processes in the manufacturing and testing of electronic circuits and on the development of fast solvers for interconnect modeling. He was awarded the title of Associate Professor by the Institute of Higher Education, Ankara, in 1993. Since 1994 he has been a Faculty Member in the Department of Electrical and Electronics Engineering of the Bilkent University, Ankara. He was a Visiting Associate Professor at the Center for Computational Electromagnetics (CCEM) of the UIUC for one semester in 1997. His research interests include the development of fast algorithms for computational electromagnetics (CEM) and the application thereof to scattering and radiation problems involving large and complicated scatterers, antennas and radars, frequency-selective surfaces, and high-speed electronic circuits. He is also interested in the theoretical and computational aspects of electromagnetic compatibility and interference analyses. Ground-penetrating radars and other subsurface-scattering applications are also among his current research interests.

Dr. Gürel is currently serving as the chairman of the AP/ED/MTT chapter of the IEEE Turkey section. 


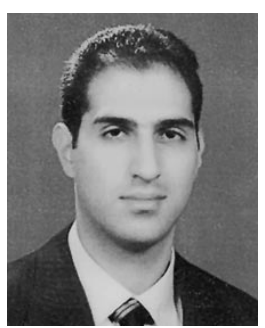

Uğur Oğuz was born in Ankara, Turkey, in 1973. He received the B.Sc. and M.Sc. degrees, both in electrical engineering, from Bilkent University, Ankara, Turkey, in 1994 and 1997, respectively.

From August 1997 to October 1998, he served in the Turkish Army, working as a Database Manager. Since November 1998 he has been a Research Engineer in the Department of Electrical and Electronics Engineering, Bilkent University, Ankara. His research interests include time-domain methods in computational electromagnetics and their applications to geophysical problems. 